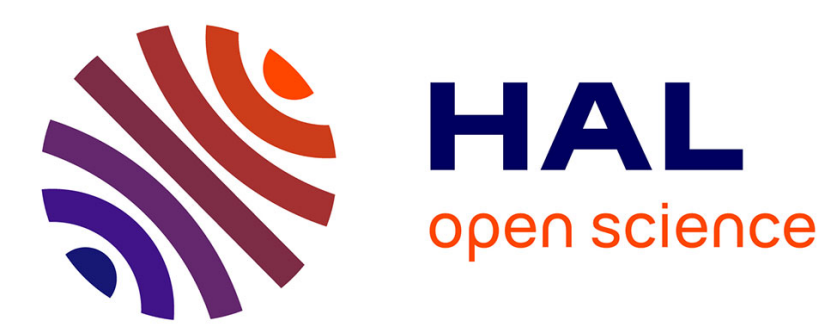

\title{
Grammaticalisation et lexicalisation : la formation d'expressions complexes
}

\author{
Sophie Prévost, Benjamin Fagard
}

\section{To cite this version:}

Sophie Prévost, Benjamin Fagard. Grammaticalisation et lexicalisation : la formation d'expressions complexes. Langue française, 2007, 156, pp.3-8. 10.3917/lf.156.0003 . halshs-01241971

\section{HAL Id: halshs-01241971 https://shs.hal.science/halshs-01241971}

Submitted on 11 Dec 2015

HAL is a multi-disciplinary open access archive for the deposit and dissemination of scientific research documents, whether they are published or not. The documents may come from teaching and research institutions in France or abroad, or from public or private research centers.
L'archive ouverte pluridisciplinaire HAL, est destinée au dépôt et à la diffusion de documents scientifiques de niveau recherche, publiés ou non, émanant des établissements d'enseignement et de recherche français ou étrangers, des laboratoires publics ou privés. 


\section{Grammaticalisation et lexicalisation : la formation d'expressions complexes}

Sophie Prévost \& Benjamin Fagard

Lattice (CNRS, ENS \& Université Paris 3 - Sorbonne nouvelle)

\section{PRÉSENTATION DU NUMÉRO}

\subsection{Introduction}

Les phénomènes de grammaticalisation et de lexicalisation ont donné lieu à des travaux désormais nombreux, et continuent à susciter régulièrement de nouvelles études, tant du côté de la description des faits relevant de l'un ou l'autre de ces processus que du point de vue théorique ${ }^{1}$.

Si la relation entre les deux est régulièrement abordée ${ }^{2}$, la nature de cette relation n'en demeure pas moins encore à approfondir, les champs respectifs de la grammaticalisation et de la lexicalisation restant sujets à discussion. Cela tient en particulier au flou qui entoure chacune de ces notions.

Ainsi, en ce qui concerne la grammaticalisation, on observe

\footnotetext{
1 Parmi les ouvrages récents, on citera en particulier Marchello-Nizia, 2006, Brinton et Traugott, 2005, Bisang, Himmelmann et Wiemer (éds), 2004, la réédition en 2003 de Hopper et Traugott (1993), Traugott et Dasher, 2002, et Wischer et Diewald (éd.), 2002.

2 Citons, entre autres, l'ouvrage désormais « ancien » (1998) de Giacalone-Ramat et Hopper (éds), The Limits of grammaticalization, avec en particulier l'article de Moreno Cabrera "On the relationships between grammaticalization and lexicalization », et parmi les travaux récents (2005), l'ouvrage de Brinton et Traugott Lexicalization and language change, et l'article de Lightfoot « Can the lexicalization / grammaticalization distinction be reconciled? ».
} 
un usage parfois abusif du terme, qui peut simplement désigner un figement relatif. Se pose aussi la question de l'extension du champ de la grammaticalisation, selon qu'elle est conçue dans une acception étroite, évolution d'une forme lexicale vers une forme grammaticale (ou évolution d'une forme grammaticale vers une forme plus grammaticale) ${ }^{3}$, ou au contraire dans une acception plus large ${ }^{4}$, évolution du discursif au morphosyntaxique, avec fixation des stratégies discursives dans les structures morpho-syntaxiques.

En ce qui concerne la lexicalisation, se pose la question de l'extension donnée au concept de « lexique », selon qu'il est entendu dans un sens étroit, ce qui donne lieu à une opposition lexical/grammatical (avec le problème de la délimitation entre les deux... ${ }^{5}$ ) ou au contraire dans un sens large, auquel cas tout est lexique, et les formes dites grammaticales font alors partie du lexique. Dans ce dernier cas, l'émergence de toute nouvelle forme, quels que soient son processus de formation et son résultat, est une lexicalisation.

Selon la perspective adoptée, lexicalisation et grammaticalisation peuvent ainsi être conçues comme des processus opposés ou non, au moins en ce qui concerne la forme « cible » résultat du processus, grammaticale ou lexicale.

Précisons que « opposition » ne signifie pas que la lexicalisation serait l' « inverse » de la grammaticalisation ${ }^{6}$. De ce point de vue, il y a un certain déséquilibre entre les deux

\footnotetext{
3 Voir en particulier Haspelmath, 1998 et 1999, Kurylowicz, 1965, Lehmann, 1982, et Traugott, 1994.

${ }^{4}$ Suite aux travaux de Givón, en particulier Givón, 1979.

5 Ce problème est d'autant plus délicat qu'on se rend compte que des catégories " grammaticales » selon la grammaire traditionnelle sont en fait « ouvertes " (en particulier les prépositions et les adverbes).

${ }^{6}$ On préférera parler dans ce cas de dégrammaticalisation, ce qui ne signifie pas non plus qu’il y ait un strict retour à une forme qui aurait précédemment subi un processsus de grammaticalisation (voir à ce propos Prévost, 2003).
} 
notions : alors que la grammaticalisation implique à la fois le caractère (plus) grammatical de la forme cible, comparée à celle de départ, et la présence de certains mécanismes ${ }^{7}$, la lexicalisation tend à mettre l'accent sur la forme d'arrivée, lexicale, et accorde, nous semble-t-il, une importance moindre aux mécanismes à l'oeuvre, même si, parmi eux, la coalescence est souvent considérée comme décisive. En outre, « forme lexicale » ne signifie pas «plus lexicale », et l'on peut d'ailleurs admettre que l'idée d'une hiérarchisation n'est pas pertinente dès lors qu'il s’agit des formes lexicales : un nom est-il plus lexical qu'un verbe ? Elle ne va d'ailleurs pas non plus de soi en ce qui concerne les formes grammaticales : une conjonction est-elle plus ou moins grammaticale qu'une préposition $^{8}$ ?

L'attention moindre accordée aux mécanismes à l'œuvre dans la lexicalisation est un point important, car même si l'on envisage (comme nous le faisons ici) la lexicalisation dans un sens étroit (forme d'arrivée " lexicale ", par opposition à "grammaticale »), on constate que certains des mécanismes à l'œuvre interviennent aussi dans les processus de grammaticalisation, en particulier en ce qui concerne la perte d'autonomie syntaxique ${ }^{9}$.

Cela explique d'ailleurs que l'évolution d'une même forme puisse être analysée comme relevant de la grammaticalisation et/ou de la lexicalisation : la formation des

\footnotetext{
7 Dont le nombre et le caractère plus ou moins obligatoire restent sujet à discussion : voir Prévost, 2003.

${ }^{8}$ Voir à ce propos Amiot et De Mulder, 2002.

9 On sait de toute façon que la plupart des mécanismes qui interviennent dans la grammaticalisation ne lui sont pas spécifiques; ce qui l'est, en revanche, c'est la conjugaison d'un certain nombre d'entre eux.
} 
adverbes en -ment peut ainsi être analysée comme un cas de grammaticalisation si l'on envisage l'évolution du morphème ment, passé du statut de nom en latin à celui de suffixe en français, et comme une lexicalisation si l'on considère la formation de l'adverbe lui-même, qui constitue l'entrée d'une nouvelle forme dans le lexique. Un tel exemple illustre bien le besoin de préciser le niveau d'analyse - ainsi que les étapes du processus - auquel on se situe.

Cet aspect est un point essentiel et, nous semble-t-il, trop peu abordé dès lors que l'on envisage, en essayant de la clarifier, la relation entre grammaticalisation et lexicalisation.

L'observation de la formation de certaines expressions « complexes » permet justement, d'une part d'étudier la mise en œuvre de différents niveaux d'analyse et, ce faisant, de délimiter différentes étapes dans le processus, et d'autre part de préciser ce que l'on entend par " classes de mots », la catégorie d' «arrivée » de l'expression ne manquant pas de soulever certaines questions.

Les expressions ici rassemblées sont les suivantes : locutions prépositionnelles (par ex. en ancien français a chief de « au bout de »), locutions verbales (par ex. prendre garde), adverbiaux modalisateurs (par ex. à la vérité) et marqueurs de topicalisation (par ex. en ce qui concerne / à propos de). Elles sont composées, pour la plupart, de formes lexicales et grammaticales, mais aussi, pour les locutions verbales, de formes seulement lexicales.

Ces expressions ont en commun que leur formation correspond à un mouvement vers une plus grande coalescence entre des formes simples, et à la diminution progressive, voire la perte totale, de leur autonomie, avec dans certains cas 
émergence à l'issue du processus d'une nouvelle expression complexe.

Il est par ailleurs intéressant de constater que la formation de certaines de ces expressions (marqueurs de topicalisation et adverbiaux modalisateurs) présente des mouvements, sinon opposés, au moins divergents. En effet, alors que la formation de l'expression elle-même correspond, nous l'avons dit, à une perte d'autonomie de ses différentes composantes, la relation de l'expression avec le reste de l'énoncé ne traduit pas un mouvement d'intégration croissante, mais au contraire un mouvement vers une plus grande indépendance sur les plans syntaxique et sémantique, dans lequel on retrouve les différentes étapes postulées par Traugott (1982) dans le mouvement de la grammaticalisation, en particulier la première (fonction propositionnelle) et la troisième (fonction expressive). Un tel constat souligne à nouveau la nécessaire prise en compte de différents niveaux d'analyse, et de manière assez complexe puisque la distinction de niveaux dont il est ici question est d'un autre ordre que celle précédemment évoquée, à savoir entre les mécanismes de formation et la catégorie de la forme résultante.

La question de la catégorie de la forme d'arrivée, en soi, mérite assurément d'être approfondie, plusieurs aspects restant peu clairs et sujets à discussion.

En particulier, on peut s'interroger sur le statut et donc le classement des expressions complexes, et cela à différents égards : sont-elles assimilables à des catégories déjà existantes ? Cela semble bien difficile à admettre en ce qui concerne les marqueurs de topicalisation ainsi que les adverbiaux modalisateurs. Pour ces expressions se pose en 
outre la question de leur positionnement sur la chaîne du grammatical au lexical (ou du lexical au grammatical d'ailleurs). On peut aussi s'interroger, en des termes différents, à propos des locutions verbales et prépositionnelles comparées aux verbes et prépositions simples existant.

Les questions liées à la notion de catégorie ou de «classe de mots » sont nombreuses... On voit donc que l'examen des différentes expressions ici envisagées permet, d'une part, de préciser leur processus de formation, encore insuffisamment décrit, et, d'autre part, d'aborder plusieurs points qui permettent de clarifier les relations entre grammaticalisation et lexicalisation, en particulier la distinction de différents niveaux d'analyse, et les glissements entre classes de mots.

\subsection{Présentation des articles}

Les études ici rassemblées portent toutes sur des phénomènes d'évolution linguistique s'étant produits dans la diachronie du français, de ses stades les plus anciens jusqu'à la langue classique et moderne. Elles s'appuient, pour ce faire, sur le dépouillement de corpus numérisés importants, mettant à profit les bases de données mises au point par diverses équipes de recherche : la Base du Français Médiéval (ICAR-UMR 5191, ENS-LSH), la Base textuelle du moyen français et Frantext (ATILF-UMR 7118).

Le premier article de ce numéro porte sur les prépositions complexes (aussi appelées locutions prépositionnelles) : Benjamin Fagard et Walter De Mulder s'attachent à la description de ces constructions, qui posent problème dans la mesure où elles associent une fonction syntaxique proche de celle des prépositions simples à une forme qui n’est 
généralement pas figée. Deux questions se posent donc : comment ces constructions émergent-elles en diachronie ? Faut-il les considérer comme une sous-catégorie des prépositions simples ? Les auteurs, partant des critères énoncés par d'autres linguistes pour le français mais aussi l'espagnol, l'allemand et l'anglais, analysent l'émergence de quelques constructions complexes formées sur base nominale en ancien français : a chief de "au bout de ", a hore de "à l'heure de », por l'amor de "pour l'amour de », au lieu de et audessus de. Ils concluent que ces constructions sont le résultat d'un processus de lexicalisation, et qu'elles peuvent dans un deuxième temps se grammaticaliser en prépositions simples, comme le montre l'exemple de du côté de > côté en français moderne.

Deux articles portent sur le figement des locutions verbales, et en particulier des structures à verbe support. Olivier Bertrand étudie les constructions formées sur le nom garde en ancien français, opposant en particulier prendre garde et avoir (la/bone) garde, tandis qu'André Valli étudie en moyen français un ensemble d'expressions plus large, incluant les verbes faire, avoir, donner et estre, ainsi que la construction il y $a$, associés à divers noms. Dans ces articles, Olivier Bertrand et André Valli discutent certaines théories récentes sur l'emploi de l'article zéro comme critère de figement, et s'appuient chacun sur un corpus de français médiéval pour analyser dans le détail le degré de liberté des différentes constructions prises en compte, en se fondant notamment sur le critère sémantique de l'opacité. Ils concluent au caractère non-figé de la plupart des expressions étudiées, et à la nécessité de combiner les deux critères (emploi de l'article zéro et figement sémantique) pour juger du degré de figement 
d'une construction.

Deux articles portent sur la formation et le fonctionnement de certains adverbiaux modalisateurs. L'article de Corinne Féron porte sur les modalisateurs en pour $N$ dans la diachronie du français : pour voir, pour vrai, pour vérité et pour certain. L'auteur souligne l'existence de trois structures majeures : pour $N$, pour dire (le/au) $N$ et pour de $N$, et étudie l'émergence de la première (pour $N$ ) à partir de constructions du type tenir/dire pour vrai/vérité... que P. À partir d'une étude sur corpus en ancien et moyen français, elle examine pour les structures pour $N$ la question du passage d'attribut de l'objet à adverbial modalisateur, et montre qu'on ne peut finalement le considérer comme un cas de grammaticalisation au sens strict.

Dans leur article, Bernard Combettes et Annie Kuyumcuyan, qui s'appuient aussi sur les constructions modalisatrices en vérité, analysent l'importance de la variation en / à la (vérite), qui caractérise cette construction et la différencie d'autres constructions pourtant proches : en toute franchise, en un mot, en bref, en toute amitié (*à toute franchise, etc.). Les auteurs étudient en détail le contexte d'émergence de ces constructions (aux $\mathrm{XV}^{\mathrm{e}}-\mathrm{XVII}{ }^{\mathrm{e}}$ siècles), ainsi que leur degré de figement. Ils montrent qu'il y a coexistence partielle des différents emplois, et en particulier que les emplois énonciatifs semblent se développer en même temps que ceux de type textuel.

Enfin, deux articles portent sur l'émergence des topicalisateurs. Dans son article, Bernard Combettes analyse en détail l'émergence des constructions en / pour ce qui 
concerne, en / pour ce qui regarde, et en / pour ce qui touche, qui sont passées d'emplois liés à des emplois en position détachée, avec, à ce stade, une réanalyse en topicalisateurs. L'auteur étudie en particulier les contextes de réanalyse de ces constructions, et s'appuie pour décrire cette évolution sur deux critères, le figement morpho-syntaxique et l'articulation discursive entre topique et commentaire. Il montre que l'alternance de en et pour à la tête de ces constructions est significative, pour fonctionnant comme la forme non marquée puisqu'elle recouvre toute la gamme des emplois, tandis que les constructions introduites par en sont globalement limitées aux emplois intraprédicatifs postverbaux.

Sophie Prévost retrace dans son article l'émergence et l'évolution des expressions à propos de $X$, à ce propos et à propos. L'étude sur corpus permet à l'auteur de montrer la spécificité de chacune de ces constructions, qui diffèrent par leur date d'apparition et leur fréquence, ainsi que par leur fonctionnement. Elle montre que toutes ont développé, à partir d'emplois pré- et/ou postverbaux plus ou moins liés, des emplois non liés relevant des plans textuel et énonciatif (marqueurs énonciatifs pour à ce propos et à propos, et topicalisateur pour à propos de $X$ ), le processus s’accompagnant de certains mécanismes qui en font une évolution typique de la grammaticalisation.

\section{Bibliographie}

Amiot D. \& De Mulder W. (2002), « De l'adverbe au préfixe en passant par la préposition: un phénomène de grammaticalisation ? », Lingvisticae Investigationes 25/2, 247-273.

Bisang W., Himmelmann N. \& Wiemer B. (éds). (2004), What makes grammaticalization?, Berlin / New York : Mouton de Gruyter.

Brinton L. \& Traugott E. (2005), Lexicalization and language change, 
Cambridge : Cambridge University Press.

Giacalone Ramat A. \& Hopper P. (éds). (1998), The Limits of grammaticalization, Amsterdam : Benjamins.

Givón T. (1979), Understanding Grammar, New York, Academic Press.

Haspelmath M. (1998), "Does grammaticalization need reanalysis? ", Studies in Language 22, 49-85.

Haspelmath M. (1999), "Why is grammaticalization irreversible? ", Linguistics 37, 1043-1068.

Heine B., Claudi U. \& Hünnemeyer F. (1991), Grammaticalization: a conceptual framework, Chicago, Chicago University Press.

Hopper P. \& Traugott E. (2003), Grammaticalization, Cambridge : Cambridge University Press.

Kuryłowicz J. (1975) [1965], " The evolution of grammatical categories », Diogenes 51 (1965), 55-71, réimpr. Esquisses linguistiques, vol II, München : Fink (1975), 38-54.

Lehmann C. (1995) [1982], Thoughts on grammaticalization, München : Lincom Europa.

Lightfoot D. (2005), " Can the lexicalization / grammaticalization distinction be reconciled? », Studies in language 29/3, 583-615.

Marchello-Nizia C. (2006), Grammaticalisation et changement linguistique, Bruxelles : De Boeck.

Prévost S. (2003), « La grammaticalisation : unidirectionnalité et statut », Le Français Moderne 71/2, 144-166.

Prévost S. (2006), « Grammaticalisation, lexicalisation et dégrammaticalisation : des relations complexes », Cahiers de Praxématique 46, 121-139.

Ramat P. (1992), " Thoughts on degrammaticalization », Linguistics 30, 549-560.

Traugott E. (1982), « From propositional to textual and expressive meanings : some semantic-pragmatic aspects of grammaticalization », in Perspectives on Historical Linguistics, Lehmann W. \& Malkiel Y. (éds), Amsterdam : Benjamins, 245-271.

Traugott E. (1994), « Grammaticalization and lexicalization », Encyclopedia of Language and Linguistics, Asher R. \& Simpson J. (éds), vol 3, Oxford : Pergamon, 1481-1486.

Traugott E. \& Heine B. (éds) (1991), Approaches to grammaticalization, Amsterdam : Benjamins, 2 vol.

Traugott E. \& Dasher R. (2002), Regularity in semantic change, Cambridge : Cambridge University Press.

Wischer I. \& Diewald G. (éds) (2002), New reflections on grammaticalization, Amsterdam/Philadelphia : Benjamins. 